\title{
STELLAR CONTENT OF THE GIANT HII REGION NGC 604
}

\author{
Bernard Debray \\ Astrophysics Division, Space Science Department of ESA \\ ESTEC, Postbus 299 \\ 2200 AG Noordwijk \\ The Netherlands
}

$B V R I$ CCD frames of NGC 604, the brightest HII region in the nearby spiral galaxy M33, have been obtained at the Nordic Optical Telescope (Canaries Islands). The seeing was $0.6^{\prime \prime}$ which corresponds to $\sim 2$ pc. at the distance of M33. The magnitude of the stars were obtained using the Capella stellar photometry package (Debray et al., 1989).

The $V / B-V$ diagram (Fig. 1) exhibits a narrow and well defined plume of blue supergiants and a much more sparsely populated plume of red stars. The presence of red supergiants confirms the statement by Israel et al. (1990) based on the strong CO absorption seen in their infrared spectra, of the presence of $M$ supergiants in the nebulae together with the hot ionizing stars.

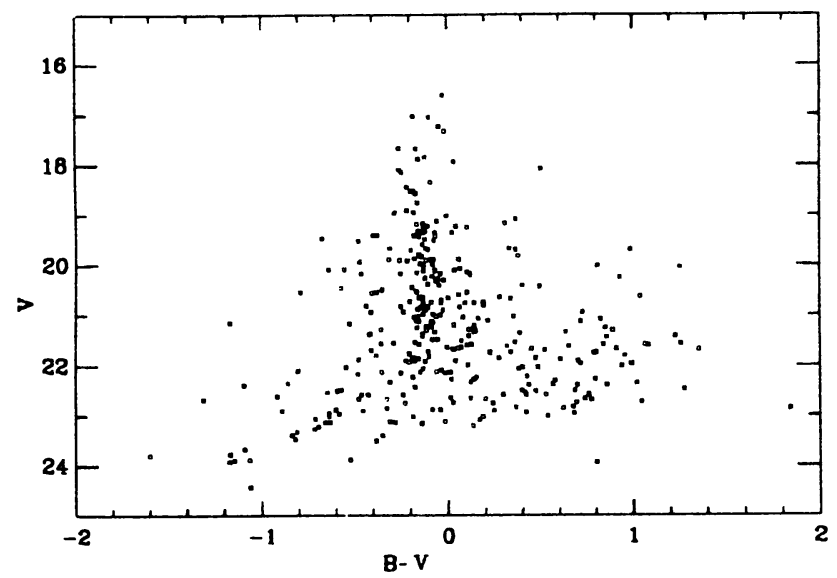

Figure 1. $V / B-V$ diagram of NGC 604.

NGC 604 contains three Wolf-Rayet stars which were once thought to be superluminous star candidates, similarly to R136a, the central object of 30 Doradus. This was first supported by Benvenuti, d'Odorico and Dumontel (1979) and then by Conti and Massey (CM, 1981) and Massey and Hutchings (1983) from IUE observations. The good seeing of the present frames points out clearly that all the bright knots inside the central core if the nebula are groups of at least a few stars (Fig. 2) as already thought by d'Odorico and Rosa (1982).

Taking the values for extinction given by Conti and Massey (1981), and assuming a distance modulus of 24.3 for M33 (Madore et al., 1985), one gets the following $M_{1}$. magnitudes for the Wolf-Rayet stars : CM11 : -7.9 (instead of -9.1); CM12 : -7.7 (instea.d of -9.5) ; CM13 : -7.4 (instead of -8.7), taking into account the different distance modulus 
used in CM. These values are now situated in the upper tail of visual magnitudes of WNL stars (Conti, 1986). But the presence of several stellar components for each object within 2 parsecs cannot be excluded.

Similar studies are planned for other giant HII regions in M33 as well as high spatial resolution narrow band imaging to investigate further their WR content.

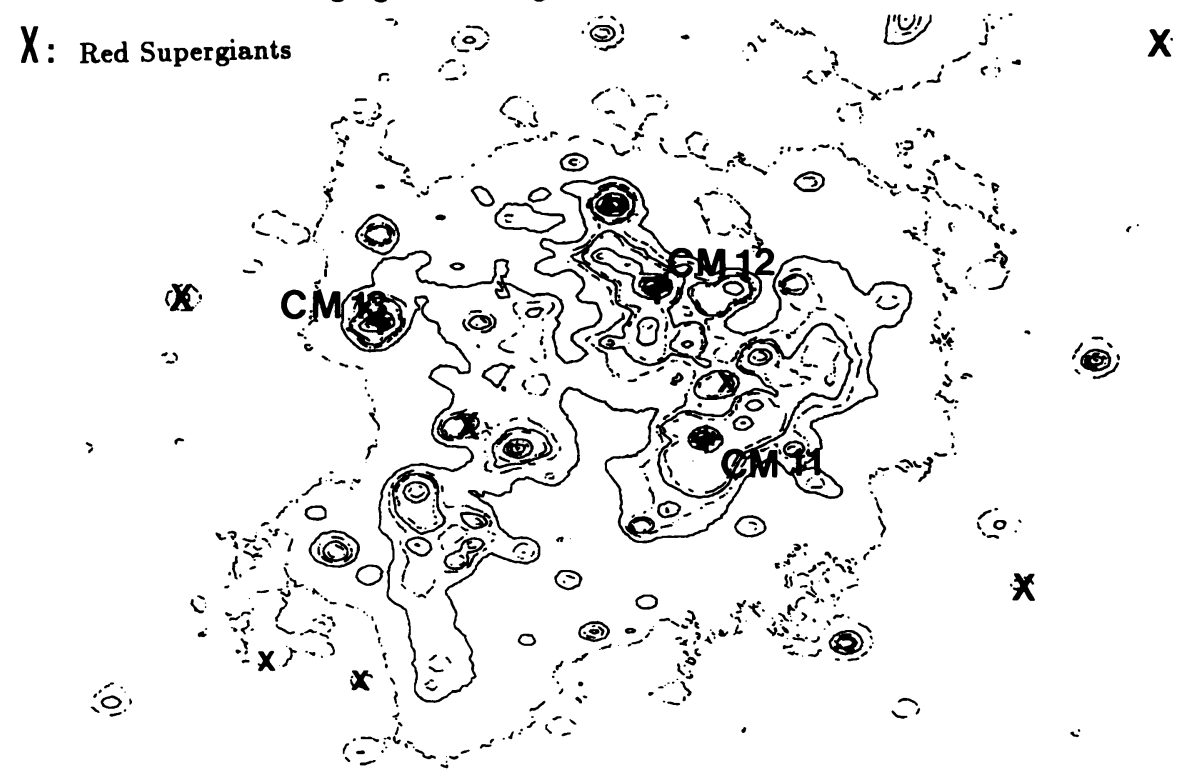

Figure 2. Isophotes of the central part of NGC 604 in V. One can see for instance clearly that CM13 is made of several components.

\section{References}

Benvenuti, P., d'Odorico, S., Dumontel, M. (1979) Astrophysics and Space Science 66, 39 Conti, P.S., Massey, P. (1981) Astrophys. Journal 249, 471

Conti, P.S. (1986) in IAU Symposium 116, Luminous Stars and Associations in Galaxies, pp.199-214

Debray, B., Llebaria, A., Dubout-Crillon, R., Petit, M. (1989) Proceedings of the 1st ESO/ST-ECF Data Analysis Workshop, P. Grosbøl, F. Murtagh and R.H. Warmels. (Eds.), 189

D'Odorico, S., Rosa, M. (1981) Proceedings of the ESO Workshop on "The Most Massive Stars", 191

Israel, F.P., Hawarden, T.G., Geballe, T.R., Wade, R. (1990) Monthly Notices Roy. Astron. Soc. 242, 471

Massey, P., Conti, P.S. (1983) Astrophys. Journal 273, 576

Massey, P., Hutchings, J.B. (1983) Astrophys. Journal 275, 578

Madore, B.F., McAlary, C.W., McLaren, R.A., Welch, D.L., Neugebauer, G., Matthews, K. (1985) Astrophys. Journal 294, 560 